\title{
Electrical and mechanical uncertainty study in cylindrical near field antenna measurement systems
}

\author{
S. Burgos*, F. Martín, M. Sierra-Castañer, J.L. Besada \\ Grupo de Radiación, Dpto. Señales, Sistemas y Radiocomunicaciones, \\ Universidad Politécnica Madrid, 28040 Madrid, Spain \\ E-mail: sarab@gr.ssr.upm.es
}

\section{Introduction}

In order to evaluate how mechanical or electrical errors may affect in the final results (i.e. radiation patterns, directivity, side lobe levels (SLL), beam width, maximum and null position...), an error simulator based on virtual acquisitions of the measurement of the radiation characteristics in a cylindrical near-field facility has been implemented [1], [2]. In this case, the Antenna Under Test (AUT) is modelled as an array of vertical dipoles and the probe is assumed to be a corrugated horn antenna. This tool allows simulating an acquisition containing mechanical errors - deterministic and random errors in the $x$-, $y$ and $z$-position - and also electrical inaccuracies - such as phase errors or noise - . Then, after a near-to-far-field transformation [3], by comparing the results obtained in the ideal case and when including errors, the deviation produced can be estimated. As a result, through virtual simulations, it is possible to determine if the measurement accuracy requirements can be satisfied or not and the effect of the errors on the measurement results can be checked. This paper describes the error simulator implemented and the results achieved for some of the error sources considered for an L-band RADAR antennas in a 15 meters cylindrical near field system.

\section{Description of the error simulator for the inaccuracies evaluation}

In this case, since the system where this work is applied is an outdoor system, there are some error sources more relevant than the others. Actually, the effects of the wind for the probe positioning and the temperature changes that affect the phase response of the cables are the ones to be considered. Thus, the strategy adopted to evaluate the sources of error is to simulate these deviations and to examine the influence that they have in the final results. This procedure starts with the modelling of the transmitting and receiving antennas. Then, the near-to-far-field transformation is applied to obtain the far-field radiation patterns. So, to evaluate how errors could affect the final results, a model of the antennas and a simulation of the acquisition process including errors has been performed. Finally, the simulator compares the outcomes achieved from the reference data (i.e. the array infinite far-field) with the ones including the deviations.

The received field in each point of the grid was calculated taking into account the field radiated by all the dipoles modified by the probe pattern. The field from a dipole in each point of the grid is given by the sum of three spherical waves [4]. The probe is an ideal conical corrugated horn characterized by the calculated radiation pattern of the main planes. For this investigation two antennas are considered. In the first analysis, the AUT is 1.44 meters long and 1.04 meters high (antenna 1). In the second study, the AUT evaluated is bigger: 5.3 meters by 2.1 meters (antenna 2). In both cases, the probe was modelled as an ideal horn (keeping $\mu= \pm 1$ ). Besides, the AUT radiating elements considered are vertical $\lambda / 2$ dipoles over a ground plane at a distance equal to $\lambda / 4$, and assumed to be infinite, so "Image Theory" can be applied. In addition, a uniform column and row excitation in amplitude and phase is considered, the distance from AUT to probe is 5 meters, the vertical path of the probe is 15 meters and the frequency selected is $1215 \mathrm{MHz}$. 


\section{Error simulator for computational, mechanical and electrical errors Computational errors}

To validate the software and checking the computational errors of the algorithm, the array infinite far-field of the antenna (product of factor array by the radiation element pattern) is compared with the far-field calculated through the cylindrical near-field acquisition. This tool was also useful for testing the elevation validity range of the near-to-far-field transformation. The next figures show the results achieved with the bigger antenna:

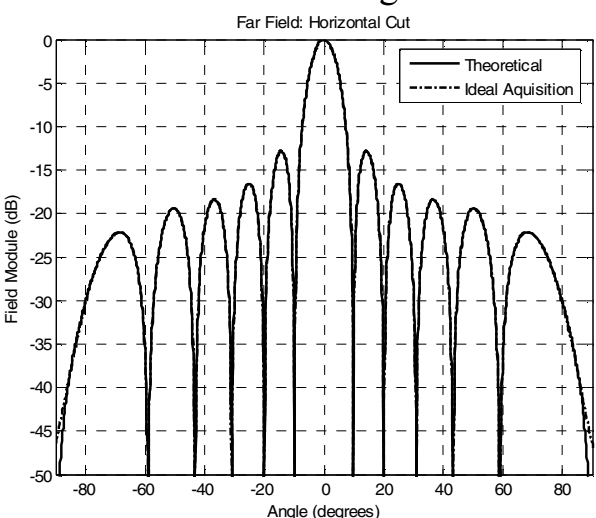

Figure 1: Horizontal cut: Theoretical versus Ideal Acquisition

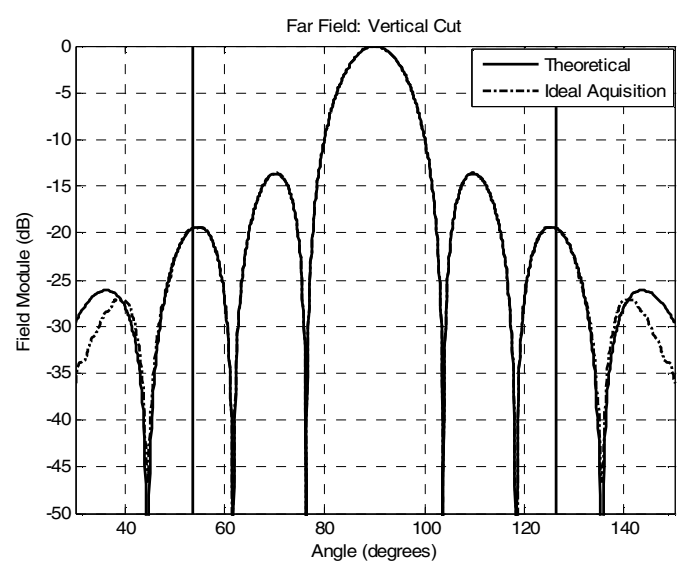

Figure 2: Vertical cut: Theoretical versus Ideal Acquisition

The diagrams obtained showed a very good agreement between the theoretical far field and the computed field and the validity of the angular margin of the measurement was confirmed.

\section{Pointing errors}

There are two different sources of deterministic errors that affect the AUT pointing: the axis non parallelism and the determination of the zero position of the azimuthal direction (in this case, the random errors caused by the wind effect are not considered). Both inaccuracies are directly translated to the error pointing, and they could be evaluated and corrected to minimize them. While the axis non parallelism can be measured with an optical procedure (i.e. laser tracker), the zero position of the azimuthal direction depends on the RADAR positioner encoder and the triggering of the vector network analyzer that can be either calculated or measured. Therefore, both errors can be compensated with a rotation of the electric field [5], and as a result they are omitted for this study.

\section{Mechanical errors in positioning system}

The positioning errors in $x$ - and $y$-direction can be very important because of the windy outdoor conditions. To evaluate the effects of the mechanical errors on the outcomes, some simulations were developed including systematic and random errors in each sample in $x, y$ and $z$ directions. While the origin of the errors in $x$ - and $y$-directions is the wind, in the $\mathrm{z}$ direction is the mechanical system of the measurement tower. Besides, the simulations were carried out with peak to peak error amplitudes in each sample from $\pm 0.05 \lambda$ to $\pm 0.2 \lambda$. From the diagrams acquired, it was clearly seen the noticeable influence of the errors on the main planes of the far-field radiation pattern. In addition, since the directivity is one of the most characteristic figures-of-merit that can describe the behaviour of an antenna, a detailed investigation has been carried out to evaluate how the mechanical errors may influence the directivity. In the simulations performed, the directivity was calculated when including a sinusoidal systematic error in the $x$-probe, a uniform random error in the $x$-probe, $y$-probe or in the $z$-probe. 
It is worthwhile noting that for the uniform random error in $x$-, $y$ - and $z$-probe the mean and standard deviation of the error introduced in the directivity were achievable. From the results, it could be noticed that as expected the magnitude of the error in the directivity increase while augmenting the error introduced in the acquisition process.

\section{Phase errors}

In near field antenna measurement systems the phase errors can be caused by temperature variations during the acquisition process. The study with the smaller antenna (antenna 1) establishes the influence that this inaccuracy may induce on the radiation pattern and directivity. As a representative example of the results achieved, Figure 3 illustrates the effect of the random phase error in the directivity when increasing the error magnitude. The line shows the average values, the dashed line the directivity without error and the triangles show the result for each individual simulation. In this case, a variable number of simulations (from 3 to 8 ) were carried out. Since several simulations were performed for each phase error, the mean and the standard deviations $(\sigma)$ of the error in the directivity were calculated, as Figure 4 shows.

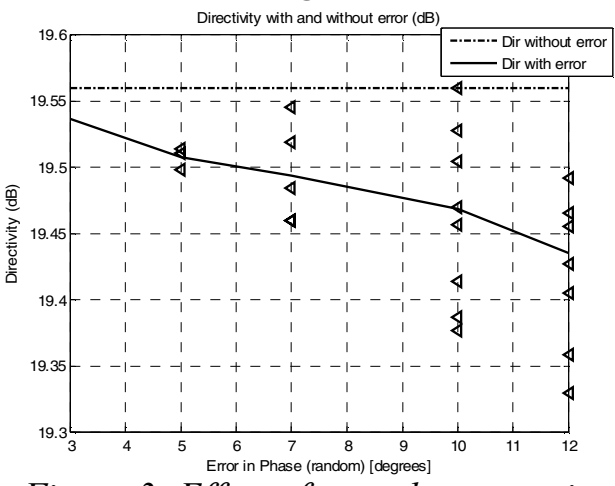

Figure 3: Effect of a random error in

Phase on the directivity

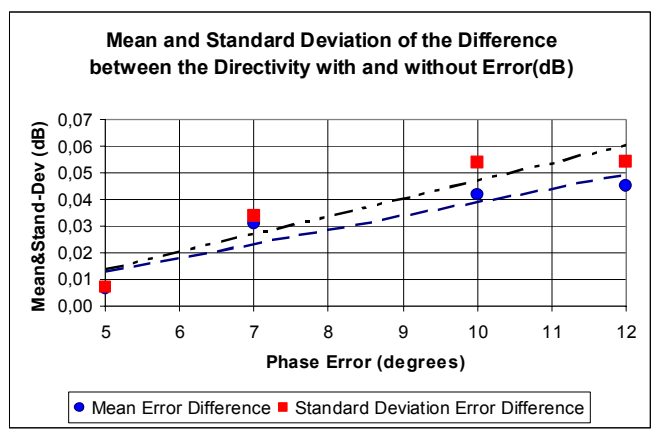

Figure 4: Mean and $\sigma$ of the difference between the directivity with and without random phase error

Once more, it could be observed, that the error in the directivity, the mean of this error and the standard deviation of this error increase linearly while the phase error introduced in the acquired field becomes larger.

\section{Errors due to the dynamic range}

Regarding the Signal to Noise Ratio (SNR) in the receiver, this magnitude can be evaluated for considering its influence on the side lobe levels. This effect was accomplished adding a random noise to each value of the acquired field. Figure 5 and Figure 6 represent the results achieved with antenna 1:

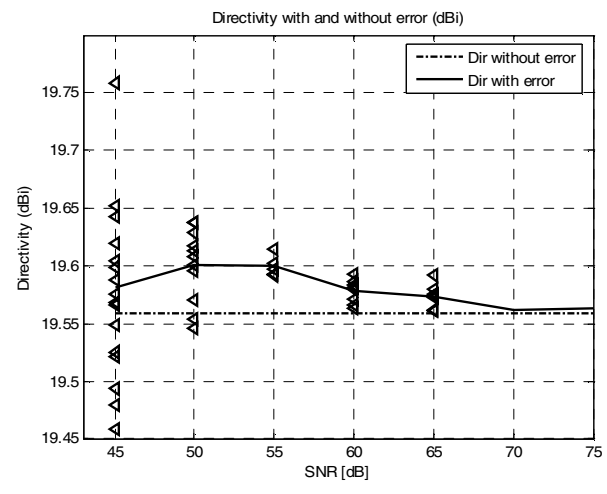

Figure 5: Effect of the noise on the directivity

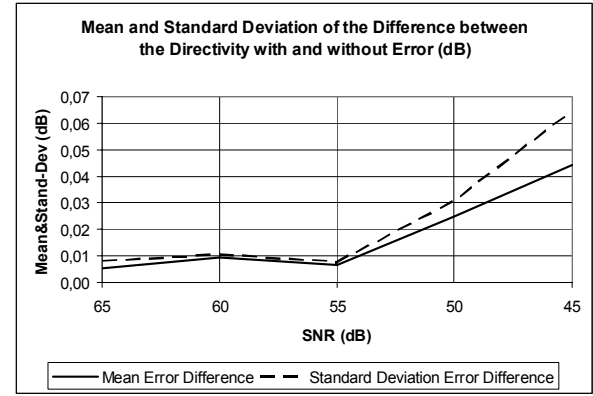

Figure 6: Mean and $\sigma$ of the difference between the directivity with and without random error due to the noise 
Similar procedure was carried out with antenna 2, providing the following outcomes:

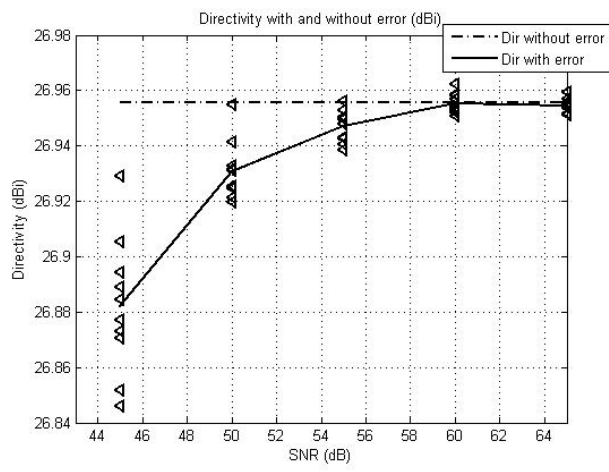

Figure 7: Effect of the noise on the directivity

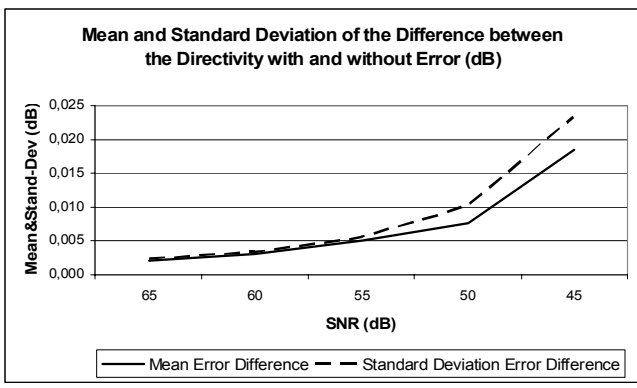

Figure 8: Mean and $\sigma$ of the difference between the directivity with and without random error due to the noise

From these outcomes, it is noteworthy that the mean and the standard deviation of the error in the directivity decrease with the improvement in the SNR. Furthermore, from this comparison, it could be noticed that the final errors on the directivity are smaller when the antenna is bigger (antenna 2).

\section{Conclusion}

A simulator has been implemented for calculating the errors in the measurement parameters of the AUT in a cylindrical near field antenna measurement system. The simulator changes the parameters of the measurement system introducing deterministic and random errors and evaluating the acquired field through the near field generated by the array of dipoles. Afterwards, it calculates the different radiation parameters and extracts the results. In addition, through the comparison between the results achieved and the infinite far-field the deviation on the final outcomes could be determined. Therefore, this simulation tool allows quantifying a priori the value of the systematic error and the uncertainty introduced in the measurement system.

\section{References:}

[1] F. Martín Jiménez, S. Burgos Martínez, M. Sierra Castañer, J.L. Besada, "Design of a Cylindrical Near Field System for RADAR antennas", Proceedings of the $1^{\text {st }}$ EuCap Conference, Nize, November 2006.

[2] S. Burgos Martínez, F. Martín Jiménez, M. Sierra Castañer, J.L. Besada, "Error Estimator in Cylindrical Near Field System for large RADAR antennas", Proceedings of the $1^{\text {st }}$ EuCap Conference, Nize, November 2006.

[3] W. M. Leach, Jr., D. T. Paris, "Probe Compensated Near-Field Measurements on a Cylinder", IEEE Transactions on Antennas and Propagation, Vol. AP-21, No.4, pp. 435-445, July 1973.

[4] Robert S. Elliot, “Antenna Theory and Design", Ed. Prentice-Hall, Inc., Englewood Cliffs, New Jersey.

[5] Y. Rahmat-Samii, "Useful Coordinate Transformations for Antenna Applications", IEEE Transactions on Antennas and Propagation, Vol. AP-27, No. 4, pp. 571-574, July 1979. 